\title{
The Use of Holographic Optical Elements in Optical Systems Sights
}

\author{
V. I. Bobrinev, Ya. A. Grad, M. S. Kovalev, P. I. Malinina*, V. V. Nikolaev, \\ S. B. Odinokov, A. B. Solomashenko and N. G. Stsepuro \\ Department of Electronics and Laser Technology, Bauman Moscow State Technical University, Moscow, \\ Russian Federation; vbobr2008@yandex.ru, y.gradinka@rambler.ru,m.s.kovalev@gmail.com, \\ pi.malinina@mail.ru,vnikolaev@hololab.ru, odinokov@bmstu.ru,art_s87@mail.ru, sng.bmstu.rl@gmail.com
}

\begin{abstract}
Objectives: This paper presents several variants of stable optical schemes of sight with Holographic Optical Element to solve a problem of changing direction of diffracted by it. Methods/Statistical Analysis: Optical scheme of holographic sight is presented where the transmission hologram together with mirror is working like the reflection one. Transmission hologram with mirror can be used to stabilize beam position for much bigger wavelength shift, than it is possible with reflection hologram. Findings: Results of calculations show that using of optical systems with a mutually inclined HOE and ADG allows reducing size of scheme with acceptable shift of image. Also it should be noted that the beneficial properties of reflection holograms can be realized using transmission holograms. Application/Improvements: Our results can be useful in devices with Holographic Optical Elements such as display systems and especially for development of holographic sight.
\end{abstract}

Keywords: Diffraction, Holography, Holographic Sight

\section{Introduction}

Holographic Optical Elements (HOE) are widely used in techniques to form wave-fronts of special kind, the typical example of such elementary HOE is Fresnel zone plate, which in simple case collimates laser light in a pointt]. One of the most famous areas of application of holograms is developing of holographic storage devices. Holograms are used as optical elements in the systems, which are used for generation of wave fronts of specific types. HOE is used in information display systems. One of practical applications of the Holographic Optical Elements is their usage for development of holographic sight ${ }^{2, \text {, }}$,

Holographic sight is used to generate the virtual image of the aiming mark on the axis, which coincides with the axis of the gun barrel and at a distance equal to the distance to the object of sighting. The luminous sign of the impact point located on the optical axis is the main element of the aiming mark. The hologram of such sign is a diffraction grating - a hologram, being recorded as the result of interference of two plane waves - plane reference wave and plane object wave (because the displayed object - point - is located at big distance).

Semiconductor lasers, which can significantly reduce weight and size of the device, are frequently used as the light source in holographic sights. A disadvantage of these lasers is changing of the wavelength of the emitted light, if the ambient temperature changes. When the temperature changes from $-50^{\circ} \mathrm{C}$ to $+50^{\circ} \mathrm{C}$ the wavelength changes from $0.62 \mu \mathrm{m}$ to $0.68 \mu \mathrm{m}$. As a result, direction of propagation of diffracted by the hologram beam is changed, causing fails of the device operation.

In this article we will discuss ways how to stabilize direction of the diffracted by HOE beam, if the laser beam wavelength is unstable (achromatization of the optical circuit), as the object of study here is considered optical circuit of the holographic sight.

In many cases HOE is recorded on media with a certain thickness of the photosensitive layer, so it is volume hologram. Operation principle of hologram is based not on laws of the geometrical optics, but on laws of diffraction and interference of light and, in particular,

${ }^{*}$ Author for correspondence 
light dispersion has a strong influence on operation of hologram.

The sight optical scheme should ensure stability of the direction of propagation of a plane wave, diffracted on the holographic elements of the circuit and carrying the virtual image of the aiming mark.

To solve the achromatization problem optical circuit of holographic sight is formed using two Holographic Optical Elements, one of them (HOE) forms a virtual image of the aiming mark, and the second - Achromatic Diffraction Grating (ADG) is used to compensate the image shift when the wavelength of the reading light beam is changed from nominal value $\lambda_{0}$.

The optical circuit of device, which can be used to provide stabilized position of the image of the aiming mark, is shown in Figure 1. There ADG is achromatic grating, HOE - Holographic Optical Element with a recorded aiming mark hologram. The angle between the planes of the ADG and the HOE is $\varphi$. Interference fringes in the two holograms are directed horizontally perpendicular to the plane of the Figure.

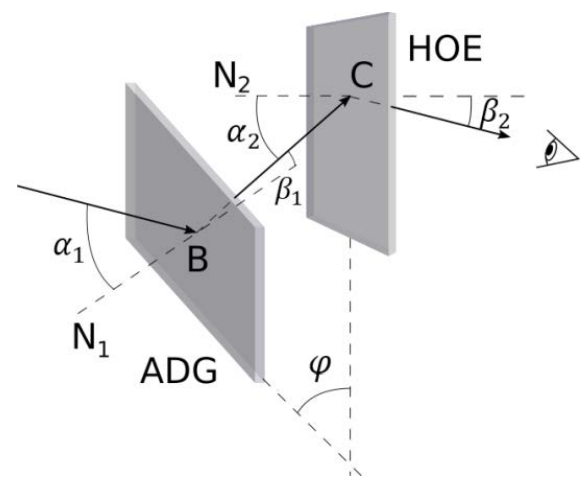

Figure 1. Principal optical scheme of holographic sight.

Dotted lines $\mathrm{N}_{1}$ and $\mathrm{N}_{2}$ are normal to surfaces of holograms, $\alpha_{1}$ - incidence angle of the laser beam to the ADG, $\beta_{1}$-deflection angle from the normal $N_{1}$ of beam, diffracted by the ADG. This beam becomes to be the reading beam for HOE, $\alpha_{2}$ - incidence angle of this beam to HOE.

$\beta_{2}$ - angle between the normal $\mathrm{N}_{2}$ and diffracted by the HOE beam, which is carrying the virtual image of the aiming mark, $\alpha_{10}, \beta_{10}, \alpha_{20}, \beta_{20}$ - the magnitude of these angles at a nominal wavelength of light $\lambda_{0}$ corresponding to the center of the tuning range, $\mathrm{d}_{1}, \mathrm{~d}_{2}$ - period of gratings recorded on ADG and HOE.

The achromatization condition of optical scheme is constant value of $\beta_{2}$ angle, when the wavelength of laser beam is changed and $\alpha_{1}$ is constant.

Light propagation of the light beams in the optical circuit on Figure 1 is described by diffraction of light by gratings formulas:

$$
\begin{aligned}
& \sin \alpha_{1}+\sin \beta_{1}=\lambda / d_{1} \\
& \sin \beta_{1}=\lambda / d_{1}-\sin \alpha_{1} \\
& \sin \alpha_{2}+\sin \beta_{2}=\lambda / d_{2} \\
& \sin \beta_{2}=\lambda / d_{2}-\sin \alpha_{2}
\end{aligned}
$$

The value of angle $\alpha_{2}$ is determined by considering the triangle $\mathrm{BCD}$ :

$90^{\circ}-\alpha_{2}+90^{\circ}+\beta_{1}+\varphi=180^{\circ}$ or $\alpha_{2}=\beta_{1}+\varphi$

Corresponding to (4) and (5), we obtain:

$\sin \beta_{2}=\lambda / d_{2}-\sin \left(\varphi+\beta_{1}\right)=\lambda / d_{2}-\sin \varphi \cos \beta_{1}-\cos \varphi \sin \beta_{1}$

If the wavelength of laser beam is changed by $\Delta \lambda$ from the nominal value $\lambda_{0}$, the angle $\beta_{1}$ is changed in accordance with Equation (1) as:

$\Delta \beta_{1} \cos \beta_{10}=\Delta \lambda / d_{1} \quad$ or $\quad \Delta \beta_{1} / \Delta \lambda=1 /\left(d_{1} \cos \beta_{10}\right)$

Assuming that $\beta_{2}$ does not change (it is possible, if the scheme is achromatized), consider the beam diffraction through the HOE with the formula (6). We will get:

$0=\frac{\Delta \lambda}{d_{2}}-\Delta \beta_{1} \cos \left(\varphi+\beta_{10}\right)$ or $\frac{1}{d_{2} \cos \left(\varphi+\beta_{10}\right)}=\frac{\Delta \beta_{1}}{\Delta \lambda}$

Joining (7) and (8), we will obtain the achromatization condition:

$d_{1}=d_{2} \cos \left(\varphi+\beta_{10}\right)=d_{2} \cos \alpha_{2}$

From (5), (6) and (9) we can get the simplest scheme of achromatization. If $\varphi=0^{\circ}$, then according to (9) there should be $\mathrm{d}_{1}=\mathrm{d}_{2}$ and $\alpha_{2}=\beta_{1}$.

Second simple option of achromatization can be realized, if $\beta_{10}=0$ and $d_{1}=d_{2} \cos \varphi$.

According to (5) we have additionally $\alpha_{20}=\varphi$. 
The third option is implemented if $\beta_{10}=-\varphi / 2$ . In this case, the diffracted by ADG beam is directed perpendicular to the bisector of the angle between ADG and HOE and the angle of incidence of the beam on the HOE at a nominal wavelength $\lambda=\lambda_{0}$ is $\alpha_{20}=\varphi+\beta_{10}=\varphi / 2$.

Let us consider in details the capabilities of these achromatization options.

\subsection{Option 1. $\left(\varphi=0^{\circ}, \mathrm{d}_{1}=\mathrm{d}_{2}\right)$}

According to the first option if $\mathrm{ADG}$ and $\mathrm{HOE}$ planes are parallel to each other and the periods of gratings, being recorded on them, are equal, the output beam direction coincides with the direction of the input laser beam and does not change when the wavelength is changed (see. Figure 2. As can be seen from the figure, the output beam is displaced parallel to itself.

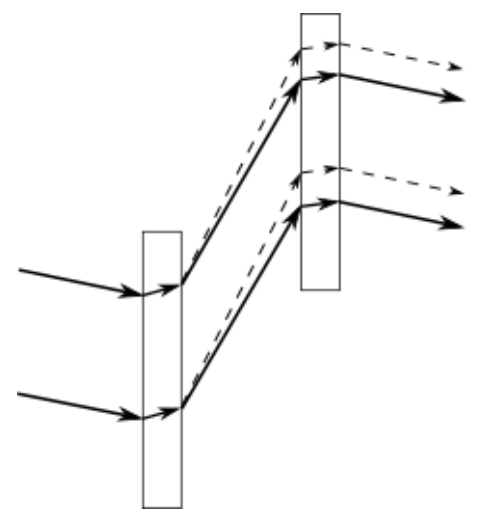

Figure 2. Scheme with parallel arrangement of ADR and $\mathrm{HOE}$ (by thick lines are presented rays passing at a nominal wavelength and by dotted lines - rays with changed wavelength).

Offset of beam parallel to itself does not affect seriously position of the image of the aiming mark, since the magnitude of this shift is much less than the dimensions of the hologram.

Additional scheme option with parallel arrangement of ADG and HOE is presented on Figure 3.

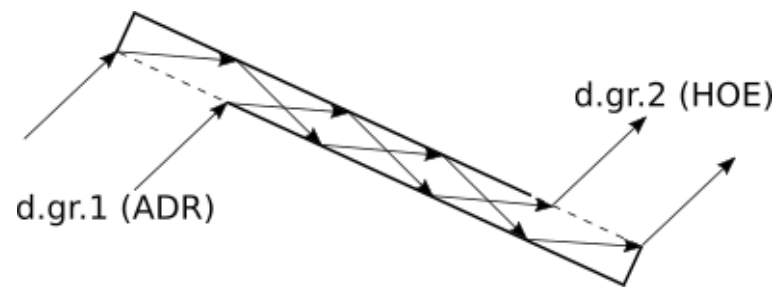

Figure 3. The waveguide achromatization scheme.
The scheme represents a glass plate on which two diffraction gratings with the same period are plotted (d.gr.1 and d.gr.2), the lines are directed perpendicular to the plane of the Figure. Gratings period is selected so that the incidence angle on the back surface of the glass plate of the wave, diffracted on a lattice 1 , is greater than the angle of total internal reflection. After a few reflections inside the plate, this wave is incident on the second diffraction grating 2 and the diffracted by this grating beam is carrying the virtual image of the aiming mark, which remains stationary when the wavelength is changed.

Optical schemes with parallel arrangement of ADR and $\mathrm{HOE}$ can operate over a wide wavelength range, but they have a significant drawback - their vertical size should be more than two times bigger than the hologram size (and accordingly the pupil of the sight).

To reduce the size of the sight let us consider possibility of other achromatization options, when $\varphi \neq 0$. In this case dependence $\beta_{2}$ of $\lambda$ cannot be completely excluded; however, in this case, there is a limited range of allowable wavelength variations.

\subsection{Option 2. $\left(\beta_{10}=0^{\circ}\right)$}

Let us consider the second achromatization option when $\beta_{10}=0^{\circ}, \alpha_{20}=\varphi$ and $\mathrm{d}_{1}=\mathrm{d}_{2} \cos \varphi$ (Figure 4). Here the thick arrows show optical path of the laser beam, being diffracted by ADR and HOE. Dotted arrow from eye shows direction to the aiming object. It is clear that this line cannot go through ADR.

If 10 , the Equation (2) will be $\sin \alpha_{1}=\lambda / \mathrm{d}_{1}$. In addition, the value of $d_{1}$ should be chosen due the condition $d_{1}=d_{2} \cos \varphi$ to stabilize the angle $\beta_{2}$.

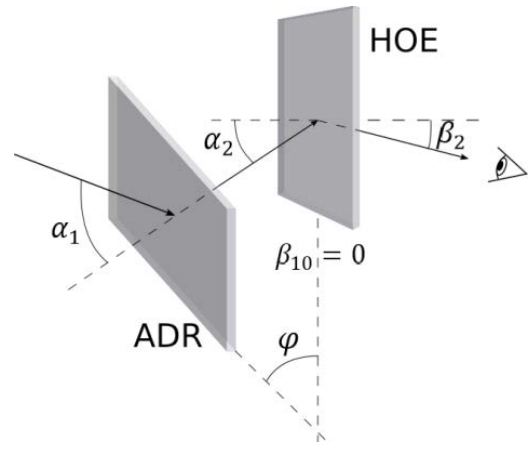

Figure 4. Holographic sight scheme with $\beta_{10}=0$

Taking into account that $\beta_{10}=0^{\circ}$, we can get $\alpha_{20}=\varphi$. 
Now to calculate dependence of $\beta_{2}$ from $\lambda$ it is necessary to choose $\alpha_{1}$ and then make calculation, using (2), (4) and (10) formulas. Let us consider the examples of optical schemes, where $\beta_{10}=0^{\circ}$.

Example 1: $\varphi=40^{\circ}, \lambda_{0}=0.65 \mu m, \beta_{10}=0^{\circ}$.

Choose $\quad \alpha_{1}=60^{\circ}$. Then $d_{1}=0,75055 \mu m$, $d_{2}=0,9798 \mu \mathrm{m}, \beta_{20}=1,1819^{\circ} \beta_{20}=1.1819^{\circ}$.

For $\beta_{2}$ dependence on $\lambda$ we have in Table 1 .

Table 1. For $\beta_{2}$ dependence on $\lambda$ (option 2, example 1)

\begin{tabular}{llllllll}
\hline$\lambda, \mu m$ & 0,60 & 0,62 & 0,64 & 0,65 & 0,66 & 0,68 & 0,70 \\
\hline$\beta_{2}, d g r$ & 1,264 & 1,211 & 1,185 & 1,182 & 1,185 & 1,211 & 1,264 \\
$\Delta \beta_{2}$, minutes & 4,9 & 1,76 & 0,2 & 0 & 0,2 & 1,76 & 4,9 \\
\hline
\end{tabular}

To have possibilities see together aiming mark and aiming object it is necessary to have $\beta_{20}$ not too small. This angle determines direction of the view and must be such that it does not go through ADG (dotted line from the eye in Figure 4). It is possible to make calculation, using $\beta_{\triangleright}$ as assigned.

Example 2: $\varphi=40^{\circ}, \lambda_{0}=0.65 \mu \mathrm{m}, \beta_{10}=0^{\circ}$

$$
\varphi=40^{\circ}, \lambda_{0}=0.6 \mu \mathrm{m}, \beta_{10}=0^{\circ}, \beta_{20}=5^{\circ} \text {. }
$$

Using (4) and (5) we have: $\mathrm{d}_{2}=0,8905 \mu \mathrm{m}$, using (10): $\mathrm{d}_{2}=0,6822 \mu \mathrm{m}, \alpha_{1}=72,34^{\circ}$

For $\beta_{2}$ dependence on $\lambda$ we obtain Table 2 .

Table 2. For $\beta_{2}$ dependence on $\lambda$ (option 2, example 2)

\begin{tabular}{|c|c|c|c|c|c|c|c|}
\hline$\lambda, \mu m$ & 0,60 & 0,62 & 0,64 & 0,65 & 0,66 & 0,68 & 0,70 \\
\hline$\beta_{2}, d g r$ & 5,0994 & 5,0358 & 5,004 & 5 & 5,004 & 5,0358 & 5,0995 \\
\hline$\Delta \beta$, minutes & 5,8 & 2,1 & 0,24 & 0 & 0,24 & 2,1 & 5,8 \\
\hline
\end{tabular}

It is seen from the table, that if the wavelength drift is $\pm 50 \mathrm{~nm}$, a beam shift is less than 6 minutes. Thus, the considered optical scheme can be used in practice.

\subsection{Option $3\left(\beta_{2}=\alpha_{20}\right)$}

Of interest is a situation where the beam, diffracted on ADG, is perpendicular to the bisector of the angle between the ADG and the HOE and, accordingly are of the same value on the angles of inclination to the ADG and HOE, when the wavelength of the reading light beam $\lambda=\lambda_{\mathbf{0}}($ Figure 5).

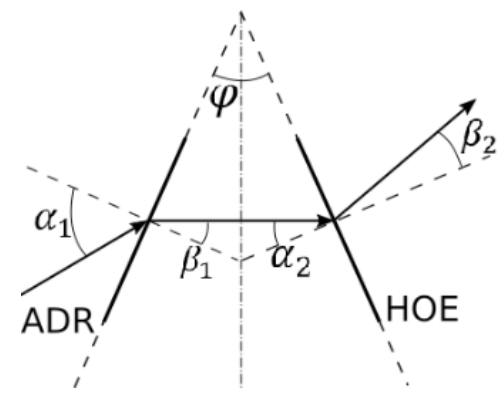

Figure 5. Optical scheme when $\beta_{10}=\alpha_{20}$ The angles $\beta_{10}=\alpha_{20}=\varphi / 2$. As a result of the formula (9), it follows that the periods of gratings, recorded on the ADG and HOE should be equal

When calculating $\beta_{2}$ dependence on the wavelength it is necessary to remember that in the scheme of Figure 5 ADG operates by formula $\sin \alpha_{1}-\sin \beta_{1}=\lambda / d_{1}$ and HOE - by $\sin \alpha_{2}-\sin \beta_{21}=\lambda / d_{2}$.

Below are presented calculations of value of $\beta_{2}$ dependence on wavelength for the scheme with the following parameters: $\varphi=40^{\circ}, \beta_{20}=15^{\circ}$.

In this case $\alpha_{\emptyset}=\emptyset^{\circ}, \mathrm{d}_{2}=\mathrm{d}_{1}=1,08182 \mu \mathrm{m}, \alpha_{1}=\emptyset, 537^{\circ}$ . For $\beta_{2}$ dependence on $\lambda$ we obtain Table 3.

A number of publications were published, where it was proposed to use reflective hologram for achromatization of optical scheme? However, the allowable shift of the laser wavelength when such holograms are used is smaller than \pm 10 nanometers due to the fact that in the reflection holograms violation of Bragg law is observed at much smaller displacements of wavelength than in the transmission holograms:.

It should be noted that the reflection holograms, which are recorded on a photographic emulsion, have another drawback. The photo emulsion is good absorber of moisture and it changes thickness of the emulsion layer, causing more strong violation of Bragg law.

It should be noted that the beneficial properties of reflection holograms can be realized using transmission holograms. To do this, it is necessary to apply a mirror coating on the back surface of the photographic plate, with recorded on it hologram, as shown in Figure 6. 


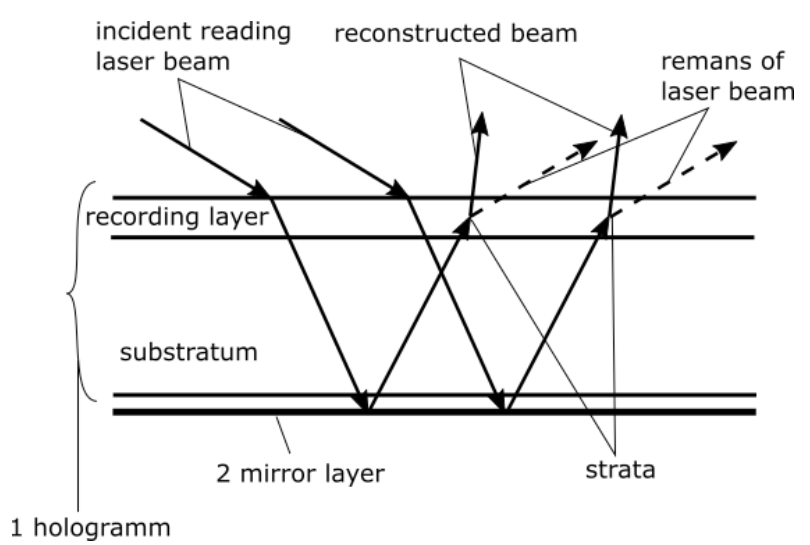

Figure 6. Transmission hologram with mirror reflector.

Hologram should be recorded in such way that the incident reading beam does not satisfy Bragg condition. This condition is satisfied for the beam passing through the hologram and reflected from the mirror 2. Reconstructed by the hologram beam is used to read the image of the aiming mark from $\mathrm{HOE}$.

Previously transmission holograms with a mirror were used to make holographic screen, which can display the stereoscopic images,

For example, there is shown on Figure 7 scheme of a holographic sight, which uses transmission hologram with a mirror.

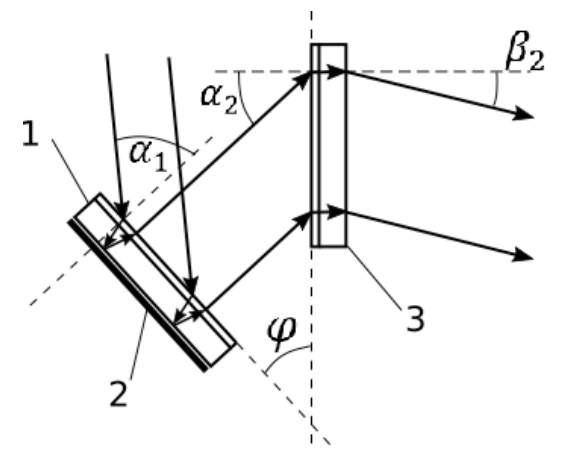

Figure 7. Optical scheme of rear sight with using transmission hologram with mirror as ADG.
Here 1 - transmission holographic diffraction grating, which one together with mirror 2 is used as achromatization element, 3 - HOE with recorded on it image of aiming point.

If $\varphi=40^{\circ}, \beta_{10}=0^{\circ}, \alpha_{1}=70^{\circ}, \lambda_{0}=0,65 \mu \mathrm{m}, \quad$ then $\mathrm{d}_{1}=0,692 \mu \mathrm{m}, \mathrm{d}_{2}=0,903 \mu \mathrm{m}, \beta_{\emptyset}=4,2^{\circ}$ Calculated stability of the reconstructed image is shown in Table 4. If laser beam wavelength is changed from $0,6 \mu \mathrm{m}$ to $0,7 \mu \mathrm{m}$, image displacement doesn't exceed 5,8 minute. Overall size of such scheme is $4,5 \times 3,6 \mathrm{~cm}$ when HOE size is 2,5 $\mathrm{cm}$.

Table 4. For $\beta_{2}$ dependence on $\lambda$

\begin{tabular}{llllllll}
\hline$\lambda, \mu m$ & $\mathbf{0 , 6 0}$ & $\mathbf{0 , 6 2}$ & $\mathbf{0 , 6 4}$ & $\mathbf{0 , 6 5}$ & $\mathbf{0 , 6 6}$ & $\mathbf{0 , 6 8}$ & $\mathbf{0 , 7 0}$ \\
\hline$\beta_{2}, d g r$ & 4,516 & 4,454 & 4,423 & 4,419 & 4,423 & 4,454 & 4,516 \\
& & & & & & & \\
$\Delta \beta_{2}$, minutes & 5,8 & 2,1 & 0,24 & 0 & 0,24 & 2,1 & 5,8 \\
\hline
\end{tabular}

Presented in Table 4 results of calculations show that using of optical systems with a mutually inclined HOE and ADG allows reducing size of scheme with acceptable shift of image.

\section{Conclusion}

There exist several options how to solve problem of image stabilization in the holographic sights by using additional transmission holographic elements for compensation of image shift.

Sight optical scheme is presented, where the transmission hologram with mirror, installed behind of hologram is used analogous to the reflection hologram.

\section{Acknowledgments}

The research was carried out at BMSTU. This work was supported by a grant \# 14.574.21.0066 (Project ID

Table 3. For $\beta$ dependence on $\lambda$ (option 3)

\begin{tabular}{lccccccc}
\hline$\lambda, \mu m$ & $\mathbf{0 , 6 0}$ & $\mathbf{0 , 6 2}$ & $\mathbf{0 , 6 4}$ & $\mathbf{0 , 6 5}$ & $\mathbf{0 , 6 6}$ & $\mathbf{0 , 6 8}$ & $\mathbf{0 , 7 0}$ \\
\hline$\beta_{2}, d g r$ & 15,050 & 15,018 & 15,002 & 15 & 15,002 & 15,017 & 15,048 \\
& 3 & 1,1 & 0,12 & 0 & 0,12 & 1,05 & 2,9 \\
\multicolumn{1}{l}{$\beta_{2}$, minutes } & & & & & & & \\
\hline
\end{tabular}


RFMEFI57414X0066) and by a grant of the main part of the state order "Organization of scientific research" and "Providing of scientific research" from the Ministry of Education and Science of the Russian Federation.

\section{References}

1. Shei HJ. The tolerance of linear scanning and the scanning angle of holographic zone plate in optical thin-film elements. Indian Journal of Science and Technology. 2012 Dec; 5(12):1-5.

2. Salakhutdinov VK. Holographic sight. Russian Federation Patent No. 2034321; 1995.

3. Kovalev MS, Kozintsev VI, Lushnikov DS, Markin VV, Odinokov SB. The method of compensation changes the position of the impact mark and a holographic collimating sight. Russian Federation Patent No. 2355989; 2007.

4. Betin AY, Dontchenko SS, Kovalev MS, Odinokov SB, Solomashenko AB, Zlokazov EY. A combination of comput- er-generated Fourier holograms and light guide substrate with diffractive optical elements for optical display and sighting system. Digital Holography and Three-Dimensional Imaging; 2015. p. 410.

5. Kolyer R, Berhart K, Lin L. Optical holography. Moscow: Mir; 1973.

6. Sieczka EJ, Tai AM, Fish RH. Low profile holographic sight and method of manufacturing same. United States Patent US2006/0164704 A1; 2006.

7. Bobrinev VI, Kovalev MS, Odinokov SB, Sagatelyan GR. Investigation of the properties of a beam reconstructed from volume Holographic Optical Elements used in optical observation devices. Russian Physics Journal. 2016; 58:1457-66.

8. Hwang SH, Bobrinev VI, Son JY. Achromatized transmission type holographic screen for a multiview stereoscopic image system. Applied Optics. 1997; 36(26):6605-10.

9. Son JY, Bobrinev VI. Method of producing a holographic projection screen for displaying a three-dimensional color images. United States Patent No US 6211977 B1; 2001. 